\title{
La maladie nodulaire cutanée des bovins I. - Situation épizootiologique actuelle en Afrique
}

\author{
par P. C. LEFEVRE $\left({ }^{*}\right)$, J. B. BONNET $\left({ }^{* *}\right)$ et B. VALLAT $\left({ }^{* *}\right)$ \\ (avec la collaboration technique de Z. GNALDAM)
}

\begin{abstract}
RÉSUMÉ
Les auteurs dressent la carte de répartition de la maladie nodulaire cutanée des bovins en Afrique et insistent sur la dynamique d'extension de cette infection.

Le cas particulier du Tchad est analysé : après l'épizootie de 1973, la maladie est actuellement enzootique.
\end{abstract}

\section{INTRODUCTION}

En 1964, K. E. WEISS, dans une monographie consacrée à la maladie nodulaire cutanée des bovins (MNCB) ou lumpy skin disease des anglo-saxons, prévoyait l'extension de cette infection (7).

En 1978, le fait est amplement confirmé et de nombreux pays du centre et de l'ouest africain sont atteints. Malgré l'extension considérable que la maladie a prise, les autorités ne considèrent pas cette infection comme grave en raison, d'une part de sa faible mortalité, et d'autre part de l'absence dans ces pays de réelles industries des cuirs et peaux.

Toutefois, la maladie sévissant en Afrique centrale et notamment au Tchad depuis plusieurs années, une mise au point était nécessaire afin de rappeler sa situation épizootiologique d'un point de vue dynamique et de tenter une estimation des pertes occasionnées.

(") Institut d'Elevage et de Médecine Vétérinaire des Pays Tropicaux. Laboratoire de Farcha, B. P. 433, N'Djamena, Tchad.

(**) Commission du Bassin du Lac Tchad, B. P. 727, N'Djamena, Tchad.

\section{SITUATION ÉPIZOOTIOLOGIQUE EN AFRIQUE}

Schématiquement, il est possible de distinguer trois périodes dans l'histoire de la MNCB :

- De 1929 à 1950. Décrite pour la première fois en Rhodésie du Nord (actuelle Zambie), la maladie est restée cantonnée dans les pays africains situés au sud de l'Equateur. Elle est signalée dans presque tous les pays du sud et du sud-ouest africain ainsi qu'à Madagascar (1 $5,7)$.

- De 1950 à 1970, la maladie s'étend vers le nord pour atteindre successivement le Zaire (1950), l'Ouganda (1956), le Kenya (1957) et le Congo (1965). Par la suite le Soudan et la R. A. U. sont à leur tour atteints (en 1970 et 1971 respectivement).

- A partir de 1970 , le MNCB infléchit sa course et gagne le Tchad et le Niger en 1973, vraisemblablement à partir du Soudan, le Nigeria en 1974 (4) et plus récemment la Côte-d'Ivoire en 1976 (6). En outre, en 1977, Le Ghana, le Liberia, le Mali et la Mauritanie ont déclaré être infectés (3). 
TABLEAU N $N^{\circ} \mathrm{I}$ - Eoyers de MACB au Tchad en 1978

\begin{tabular}{|c|c|c|c|c|}
\hline Province & Foyer & $\begin{array}{c}\text { Nombre total } \\
\text { anlmaux }\end{array}$ & $\begin{array}{l}\text { Nombre de } \\
\text { malades }\end{array}$ & Pourcentage \\
\hline Chari-Baguirmi & Kreniffe & 300 & 20 & $6-7$ \\
\hline \multirow[t]{2}{*}{ Mayo-Kebbi } & Ribao & 223 & 25 & $"$ \\
\hline & Berlian & 225 & 16 & 7 \\
\hline Moyen-Chare & Bekamba & environ 200 & 10 & 5 \\
\hline Logone & Mound ou & $?$ & $?$ & $?$ \\
\hline Total & & \pm 948 & 71 & 7,5 \\
\hline
\end{tabular}

Ainsi donc, en moins de 50 ans, la MNCB, partie du sud de l'Afrique, est remontée vers le nord puis s'est étendue d'est en ouest sur presque tout le continent africain.

Rien, à priori, ne semble pouvoir limiter sa progression si aucune mesure prophylactique n'est prise. En outre, dans les pays atteints, elle survit à l'état enzootique comme le prouve le cas du Tchad.

\section{LA MNCB AU TCHAD}

\section{Epizootie de 1973}

Son introduction au Tchad sur le cheptel pleinement réceptif a entraîné une épizootie grave. Les renseignements sont malheureusement fragmentaires quant à la répartition géographique, la maladie sinon inconnue, du moins méconnue des services vétérinaires n'ayant pas été diagnostiquée dans de nombreuses régions.

Elle a été vue, pour la première fois, semble-t-il, le long de la piste à bétail du $13^{\mathrm{e}}$ parallèle (GOURVIL, communication personnelle), voie logique de pénétration depuis le Soudan. Toutefois, PROVOST (6), donne une explication, différente : lors des transhumances vers le sud des bovins tchadiens et soudanais auraient pu entrer en contact en Empire Centrafricain et l'explosion clinique aurait alors coïncidé avec la remontée vers les pâturages de saison des pluies. Cette hypothèse a l'avantage d'expliquer la multiplicité des foyers apparus simultanément dans diverses régions.

\section{En 1978}

Bien que signalée à trois reprises en 1975 , aucun renseignement précis n'est disponible sur cette année. En revanche, en 1978, elle a été vue dans diverses 'provínces du pays à partir de la fin de la saison des pluies en septembreoctobre.

Il faut noter que les provinces où la maladie a été signalée ne représentent qu'une faible fraction de la superficie du territoire tchadien et que ce ne sont pas les régions traditionnelles d'élevage. La grande majorité des troupeaux (pour la plupart nomades) ne sont pas surveillés par les services vétérinaires.

Autant l'épizootie de 1973 avait été grave puisque, dans la seule région de l'Assale, sur 100000 têtes de bovins 80 p. 100 des animaux avaient été atteints entre la fin juillet et la miseptembre, autant en 1975 la morbidité s'est révélée faible comme le montre le tableau cidessus.

\section{CONCLUSIONS}

L'extension de la maladie nodulaire cutanée des bovins est considérable et son importance ne doit pas être sous-estimée sous prétexte qu'elle ne présente qu'un taux de mortalité quasiment négligeable.

Les pertes économiques dues à la maladie - tarissement des laitières, retard de croissance des jeunes, dépréciation du cuir - sont certainement importantes lors de la première épizootie, mais nettement moindre lorsque la maladie est enzootique.

Néanmoins le problème reste entier si une industrie des cuirs et peaux doit se développer. D'autre par, il apparaît que dans les pays où elle existe, il semble difficile d'en réaliser l'éradication.

En outre, cette extension, que rien ne semble pouvoir arrêter si aucune mesure prophylactique (vaccination, par exemple, ou contrôle aux frontières) n'est prise, peut très bien déborder le cadre du continent africain. 
SITUATION EPIZOOTIOLOGIQUE DE LA M.N.C.B EN AFRIQUE ET MADAGASCAR

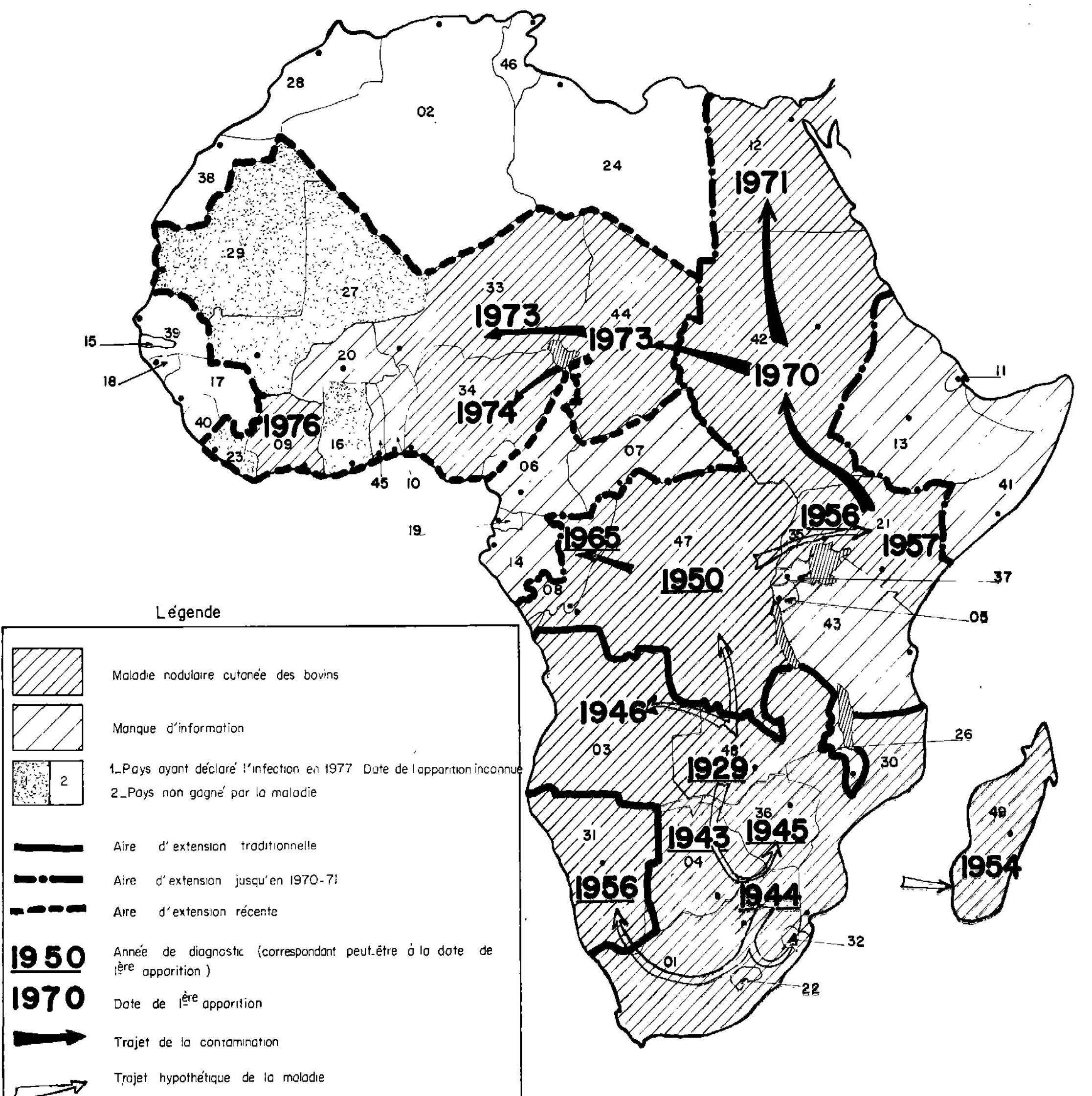

AFRIQUE DU SUD

02 ALGERI

03 ANGOLA

O4 BOTSWANA

05 BURUNDI

06 CAMEROUN

07 CENTRAFRIQUE

O8 CONGO

OP COTE D' IVOIRE

10 DAHOMEY ( BENIN)

DJIBOUTI

EGYPTE

ETHIOPIE

GABON

GAMBIE

GHANA

GUINEE CONAKRI

GUINEE BISSAU

GUINEE EQUATORIALE

HAUTE VOLTA

KENYA

LESOTHO

LIBERIA

LIBYE

MALAWI

MALII

MAROC

MAURITANIE

MOZAMBIQUE

NAMIBIE (SUD OUEST AFRICAIN)

NGWANE (SWAZILAND)

NIGER

NIGERIA

OUGANDA

RHODESIE

RUANDA

(ex) SAHARA ESPAGNOL

SENEGAL

SIERRA LEONE

SOMALIE

SOUDAN

TANZANIE

TCHAD

TOGO

TUNISIE

ZAIIRE

ZAMBIE

MADAGASCAR 


\section{REMERCIEMENTS}

Nos plus vifs remerciements vont à M. CLANET, responsable du Laboratoire de Cartographie de l'Université du Tchad, qui a eu l'obligeance de dresser la carte de répartition. Nous tenons aussi à remercier le $\mathrm{D}^{\mathbf{r}} \mathbf{K}$. E. WEISS, Directeur du Laboratoire d'Onderstepoort à qui nous devons la souche de référence.

\section{SUMMARY}

\section{Lumpy skin disease. I. - Epizootiology in Africa}

The authors draw the repartition map of lumpy skin disease in Africa with emphasis on the dynamic of extension of the disease. The case of Chad is examined : after the 1973 epizooty, the disease is now enzootic.

\section{RESUMEN}

La dermatosis nodular de los bovidos. I. - Epizootiologia en Africa

Los autores dibujan el mapa de repartición de la dermatosis nodular e insisten sobre la dinámica de extensión de dicha enfermedad. Analizán también el caso de Chad : Después de la epizootia de 1973, la dermatosis nodular está actualmente enzootica.

\section{BIBLIOGRAPHIE}

1. BOURDIN (P.). La maladie nodulaıre cutanée des bovins. Paris, Expansion scientifique française, 1971, 100 p. (Coll. Les Maladies à virus).

2. Bulletin de l'Office International des Epizooties, années 1975-1976-1977-1978.

3. F. A. O.-W. H. O.-O. I. E. Annuaire de la santé animale, 1977.

4. NAWATHE (D. R.), GIBBS (E. P. J.), OSAGBA (M. O.) et LAW MAN (M. J. P.). Lumpy skin disease in Nigeria. Trop. anim. Hith, Prod., 1978, $10: 49-54$.
5. RAMISSE (J.), SERRES (H.), RAKOTONDRAMARY (E.). Isolement à Madagascar de vırus associé à la dermatose nodulaire bovine. Rev. Elev. Méd. vét. Pays trop., 1969, 22 (3) : 357-362.

6. Rapports annuels 1973-1974-1976. N'Djaména, Tchad, Laboratoire de Farcha, I. E. M. V. T.

7. WEISS (K. E.). La dermatose nodulaire. In : Maladies nouvelles des animaux. Rome F. A. O., 1964. (Coll. Etudes agricoles F. A. O. n ${ }^{\circ}$ 61.) 